\title{
Ja zu einer zeitgemässen Fortpflanzungsmedizin
}

\section{Bruno Imthurn}

Prof. Dr. med., Vorstandsmitglied Schweizerische Gesellschaft für Gynäkologie und Geburtshilfe SGGG gynécologie suisse

Im Juni entscheidet das Stimmvolk über die Fortpflanzungsmedizin. Die Schweizerische Gesellschaft für Gynäkologie und Geburtshilfe (SGGG gynécologie suisse) setzt sich für den neuen Verfassungsartikel ein, weil damit die Präimplantationsdiagnostik als schonendste Form der Pränataldiagnostik auch in der Schweiz möglich wird und risikoreiche Mehrlingsschwangerschaften reduziert werden können.

\section{Was bisher geschah}

Im Mai 1992 wurde der heutige Artikel 119 der Bundesverfassung (BV) als Gegenvorschlag auf die «Beobachter-Initiative» von Stimmvolk und Ständen mit grosser Mehrheit angenommen. Art. 119 Abs. 2 Bst. c BV beinhaltet unter anderem folgenden entscheidenden Passus:

c. [...] es dürfen nur so viele menschliche Eizellen ausserhalb des Körpers der Frau zu Embryonen entwickelt werden, als ihr sofort eingepflanzt werden können.

Trotz der im internationalen Kontext sehr restriktiven Fassung von Artikel 119 BV wurde nur zwei Jahre später ein weiteres Begehren zum Thema eingereicht, die «Initiative für menschenwürdige Fortpflanzung». Sie forderte das komplette Verbot der assistierten Reproduktionsmedizin in der Schweiz. Diese Verbotsinitiative kam im Jahre 2000 zur Abstimmung. Mit einem überwältigenden Mehr von über 70 Prozent NeinStimmen wurde sie vom Schweizer Stimmvolk und allen Ständen wuchtig abgelehnt. An ihrer Stelle trat im Jahre 2001 der indirekte Gegenvorschlag von Bundesrat und Parlament in Kraft, das Bundesgesetz über die medizinisch unterstützte Fortpflanzung (Fortpflanzungsmedizingesetz; FMedG) [1]*.

Das im europäischen Vergleich extrem eng gefasste FMedG enthält viele bis heute gültige Verbotsbestimmungen. So sind insbesondere die Präimplantationsdiagnostik (PID) und die Kryokonservierung von Embryonen wegen Missbrauchsängsten untersagt (FMedG Art. 5 Abs. 3, Art. 17. Abs. 3). Dabei handelt es sich um die früheste Methode der Pränataldiagnostik. Bei der PID werden dem Embryo am Tag 5, also vor dem Transfer in den Uterus, Zellen des Trophektoderms -
Vorläuferzellen von Chorion und Plazenta - entnommen und genetisch untersucht. Im Unterschied zur auch in der Schweiz seit Jahrzehnten praktizierten Pränataldiagnostk steht das Paar aber bei ungünstigem Untersuchungsresultat nicht vor dem schwerwiegenden Entscheid eines Schwangerschaftsabbruchs.

Das FMedG wurde schnell vom medizinischen Fortschritt überholt. Das führte dazu, dass Paare in der Schweiz nicht mehr optimal behandelt werden konnten. Deswegen reichte der damalige Nationalrat Felix Gutzwiller die Motion «Zulassung der Präimplantationsdiagnostik» ein, die noch im selben Jahr von beiden Parlamentskammern angenommen wurde [2, 3]. Sie beauftragte den Bundesrat, die PID in der Schweiz unter vorgegebenen Rahmenbedingungen zuzulassen und das FMedG entsprechend anzupassen. Es dauerte zehn Jahre, bis Ende 2014 das Parlament eine Anpassung von Artikel BV 119 und eine Revision des FMedG beschloss und verabschiedete.

\section{Präimplantationsdiagnostik in den meisten Ländern der Europäischen Union schon seit vielen Jahren Standard}

Die European Society for Human Reproduction and Embryology (ESHRE) analysierte die Situation in Europa im Jahre 2009 [4]. Das Resultat dieser Untersuchung ergab, dass die PGD (Preimplantation Genetic Diagnosis), bei der meist bekannte Mutationen gesucht werden, schon zu dieser Zeit in 23 der damals 27 EU-Staaten zugelassen war. Es folgten Lettland, Deutschland im Jahre 2014, Kroatien mit dem Beitritt zur EU im Jahre 2013 und vor kurzem auch Österreich. 


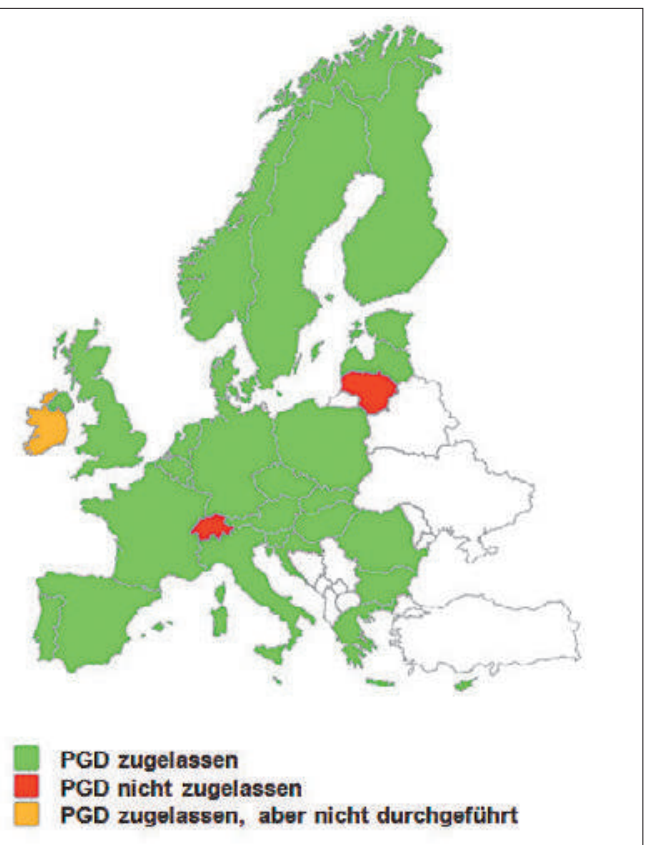

Abbildung 1: Gesetzliche Lage zur Präimplantationsdiagnostik (PGD; Preimplantation Genetic Diagnosis) in der Europäischen Union, Norwegen und der Schweiz, Stand 2015; adaptiert und aktualisiert nach [4].

Ebenso ist die PGD in Norwegen erlaubt (Abb. 1). Es haben sich somit in vielen europäischen Ländern diverse Parlamente intensiv mit der PGD befasst. Sie alle sind zum Schluss gekommen, dass diese früheste Methode der Pränataldiagnostik auch in ihren Ländern möglich sein sollte. Zur PID wird auch das PGS (Preimplantation Genetic Screening) gezählt. Dabei werden die Zellen auf das Vorhandensein von überzähligen oder fehlenden Chromosomen analysiert. Auch diese Form der PID war bereits 2009 in 20 EUStaaten erlaubt [4] und soll in der Schweiz ebenfalls zugelassen werden. Bemerkenswert ist, dass die PID in einigen europäischen Staaten schon seit über 20 Jahren praktiziert wird.

\section{Anpassung der Bundesverfassung Art. 119 an die medizinischen Bedürfnisse}

Die PID ist in der Schweiz nicht auf Verfassungs-, sondern auf Gesetzesstufe verboten (FMedG Art. 5 Abs. 3). Allerdings macht Art. 119 Abs. 2 Bst. c BV eine wirksame PID unmöglich. So schreiben Verfassung und das daraus abgeleitete FMedG vor, dass maximal drei befruchtete Eizellen zu Embryonen entwickelt werden dürfen (sogenannte 3er-Regel).

Im Durchschnitt entwickelt sich - auch bei einer natürlichen Schwangerschaft - aus sechs (!) befruchteten Eizellen nur eine einzige bis zu einem lebensfähigen Kind. Bei vielen genetischen Erkrankungen sind zudem 25 bis 50 Prozent der Embryonen von der schweren Krankheit betroffen. Mit der 3er-Begrenzung würde somit am Ende eines höchst komplexen, aufwendigen und die Paare belastenden Prozesses häufig kein Embryo zur Verfügung stehen, der von der schweren Erbkrankheit nicht betroffen ist.

Aus diesem Grund haben Bundesrat und Parlament beschlossen, diesen Verfassungsartikel den medizinischen Bedürfnissen anzupassen und folgendermassen zu ändern [5]:

c. [...] es dürfen nur so viele menschliche Eizellen ausserhalb des Körpers der Frau zu Embryonen entwickelt werden, als für die medizinisch unterstützte Fortpflanzung notwendig sind.

Wie jede Verfassungsänderung untersteht diese Anpassung dem obligatorischen Referendum und wird am kommenden 14. Juni Volk und Ständen zur Genehmigung vorgelegt.

\section{Die Revision des Fortpflanzungsmedizin- gesetzes ermöglicht eine zeitgemässe Fortpflanzungsmedizin}

Die Anpassung von Artikel 119 BV ermöglicht eine Revision des FMedG, gegen welche nach einer Annahme der Verfassungsänderung das - allerdings fakultative Referendum ergriffen werden kann [6].

Verfassungsänderung und Revision des FMedG sehen vor, die Anzahl der Embryonen, die in einem Behandlungszyklus entstehen dürfen, den medizinischen Bedürfnissen anzupassen, um a) die Erfolgschancen der betroffenen Paare zu optimieren und b) die Behandlungsrisiken für Mutter und Kind zu minimieren. Damit kann eine methodisch sinnvolle PID durchgeführt und das Mehrlingsrisiko substantiell reduziert werden.

Obschon es ausser der Schweiz mit Ausnahme von Deutschland weltweit kein Land gibt, das eine Beschränkung der Anzahl möglicher Embryonen kennt, wurde die 3er-Regel nicht gänzlich aufgehoben, sondern neu eine 12er-Regel eingeführt. Auch in Deutschland wird über die Sinnhaftigkeit der 3er-Regel diskutiert. Mit dieser neuen Obergrenze und der gleichzeitigen Zulassung der Embryokryokonservierung wird es möglich, am Tag 5 der embryonalen Entwicklung eine PID durchzuführen und zwar mit einer realistischen Chance, einen von der genetischen Krankheit nicht betroffenen Embryo zu finden. Sollte mehr als ein Embryo nicht betroffen sein, dürfte er unter der neuen Regelung für eine zweite Chance kryokonserviert und maximal zehn Jahre aufbewahrt werden. Wie bereits erwähnt, entwickelt sich im Durchschnitt nur eine von insgesamt sechs am Tag 1 befruchteten 
Eizellen zu einem lebensfähigen Kind. Dabei degeneriert natürlicherweise ein Grossteil der nicht entwicklungsfähigen Embryonen bereits sehr früh: zwischen Tag 1 und Tag 5. Es fällt damit viel leichter, mit klar definierten, morphologischen Kriterien (neu) am Tag 5 aus maximal 12 Embryonen den Embryo mit dem grössten Entwicklungspotential zu eruieren als mit unpräzisen Vorgaben (bisher) bereits am Tag 1 aus nur 3 befruchteten Eizellen. Diese Optimierung ermöglicht es, dass am Tag 5 nur noch ein Embryo transferiert werden muss - gegenüber bisher 2 bis 3 Embryonen bei vergleichbarer Schwangerschaftschance. Ein solches Vorgehen wird als elektiver Single-Embryo-Transfer (eSET) bezeichnet. Der eSET reduziert die Risiken für Mutter und Kind, da mit dem Transfer von nur noch einem Embryo am Tag 5 das Mehrlingsrisiko bei einer assistierten Kinderwunschbehandlung substantiell gesenkt werden kann. Das Hauptrisiko jeder Mehrlingsschwangerschaft ist die Frühgeburtlichkeit und damit das kindliche Sterbeund Behinderungsrisiko.

Dass mit dem eSET die Mehrlingsinzidenz markant reduziert werden kann, zeigen die Zahlen aus Schweden, das den eSET schon vor mehr als zehn Jahren einge-

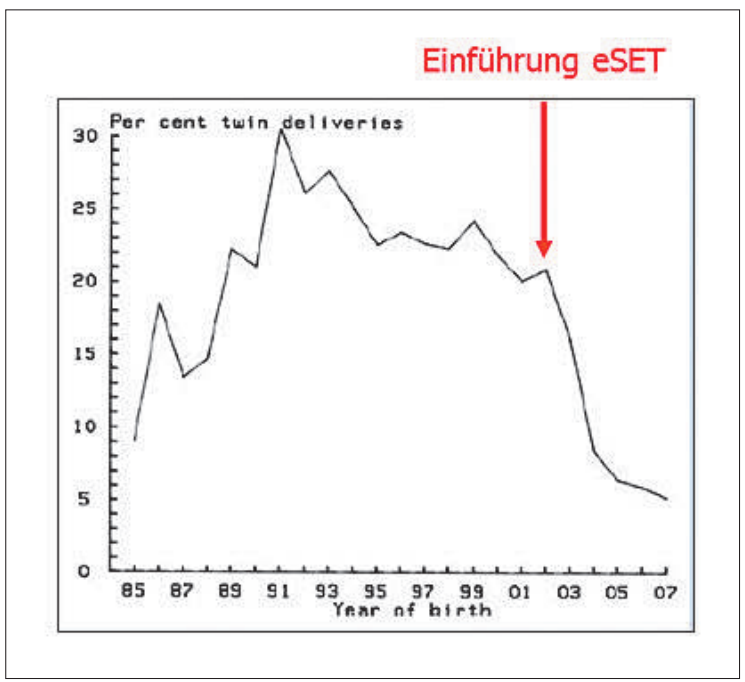

Abbildung 2: Entwicklung der Zwillingsraten in Prozent bezogen auf die Gesamtzahl aller Geburten nach assistierten Sterilitätstherapien in Schweden nach Einführung des elektiven Single-Embryo-Transfers (eSET) im Jahre 2002 [8]. führt hat. Während in der Schweiz das Mehrlingsrisiko nach einer assistierten Sterilitätstherapie gemäss Jahresstatistik von FIVNAT-CH, dem nationalen Register der Schweizer Kinderwunsch-Zentren, selbst im Jahre 2012 noch auf rund 18 Prozent verharrte [7], ist die Mehrlingsrate nach In-Vitro-Fertilisation-Behandlung (IVF) in Schweden unter zunehmender Anwendung des eSET von 2002 bis 2007 auf unter 5 Prozent gefallen (Abb. 2) [8].

Eine vorgeburtliche Untersuchung wird ohne das Dilemma des Schwangerschaftsabbruchs möglich.

Die Vorteile des eSET sind unbestritten. Bei der PID gehen die Meinungen allerdings auseinander. Die Indikationen für die PID sind im aktuellen Gesetzesvorschlag jedoch dieselben, wie sie schon seit langem in der Schweiz bei der Pränataldiagnostik nach der 11. Schwangerschaftswoche üblich und von einem Grossteil der Bevölkerung akzeptiert sind. Da die genetische Untersuchung noch vor der Übertragung eines Embryos in die Gebärmutter erfolgt, steht das Paar bei einem ungünstigen Untersuchungsresultat - im Unterschied zur bisher gängigen Pränataldiagnostik nicht vor dem Entscheid eines Schwangerschaftsabbruchs mit all seinen tragischen Konsequenzen. Deshalb befürwortet auch die Mehrheit der Nationalen Ethikkommission (NEK) die PID [9].

\section{Darum ein Ja am 14. Juni 2014}

Die SGGG gynécologie suisse setzt sich für die Anpassung von Artikel 119 BV ein, weil damit Paare, die unter einem unerfüllten Kinderwunsch leiden, in Zukunft auch in der Schweiz auf einem in Europa üblichen Stand behandelt werden können. Insbesondere kann mit dieser Verfassungsänderung (a) die Zahl der risikobehafteten Mehrlingsschwangerschaften nach Kinderwunschbehandlungen markant reduziert und (b) die PID als früheste und schonendste Form der Pränataldiagnostik auch in der Schweiz eingeführt werden. Damit wird eine vorgeburtliche Untersuchung ohne das Dilemma des Schwangerschaftsabbruchs möglich. 


\section{Referenzen}

1 Bundesversammlung der Schweizerischen Eidgenossenschaft. Bundesgesetz über die medizinisch unterstützte Fortpflanzung (Fortpflanzungsmedizingesetz, FMedG). Schweizerische Eidgenossenschaft 1998/2006;SR 810.11:1-14.

2 Gutzwiller F. Parlamentarische Initiative «Präimplantationsdiagnostik. Bewilligung». 2004;04.423.

3 Kommission für Wissenschaft, Bildung und Kultur des Nationalrats. Motion «Zulassung der Präimplantationsdiagnostik». 2004;04.3439.

4 Comparative Analysis of Medically Assisted Reproduction in the EU. MAR Study. ESHRE 2009;1-157.

5 Bundesversammlung der Schweizerischen Eidgenossenschaft. Bundesbeschluss über die Änderung der Verfassungsbestimmung zur Fortpflanzungsmedizin und Gentechnologie im Humanbereich. Schweizerische Eidgenossenschaft 2014;9675-6.
6 Bundesversammlung der Schweizerischen Eidgenossenschaft. Bundesgesetz über die medizinisch unterstützte Fortpflanzung (Fortpflanzungsmedizingesetz, FMedG). Schweizerische Eidgenossenschaft 2014;1-9.

7 Schweizerische Gesellschaft für Reproduktionsmedizin. FIVNAT-Report Cycles 2012. 2014:6

8 Källen B, Finnström O, Lindam A, Nilsson E, Nygren KG, Otterblad Olavsson P. Trends in delivery and neonatal outcome after in vitro fertilization in Sweden: data for 25 years. Hum Reprod 2010;25:1026-34.

9 Nationale Ethikkommission im Bereich Humanmedizin. Präimplantationsdiagnostik. 2005:51. 